\title{
USO DE TÉCNICAS DE MANUFATURA ENXUTA: UM ESTUDO PRELIMINAR NO SETOR AUTOMOTIVO BRASILEIRO
}

\author{
Henrique Gouvêa Quinhonero, Luan Leme Santaela, Guilherme Canuto da Silva \\ Universidade Federal do ABC (UFABC) \\ E-mails: henrique_gq@hotmail.com, luan.leme.santaela@gmail.com, \\ guilherme.canuto@ufabc.com
}

\section{RESUMO}

Este trabalho tem como objetivo identificar o uso de técnicas específicas de manufatura enxuta no setor automotivo brasileiro. Para isto um referencial teórico foi desenvolvido. Com base no referencial teórico as principais técnicas de manufatura enxuta foram identificadas. Posteriormente, uma pesquisa de campo para identificar a prática destas técnicas no setor automotivo foi realizada. Os resultados desta pesquisa de campo são mostrados e discutidos e o trabalho é encerrado com as considerações finais.

\section{INTRODUÇÃO}

A Manufatura Enxuta envolve diversas ferramentas de gestão para que a produção seja otimizada, viabilizando atingir mais facilmente objetivos de desempenho principais como qualidade, velocidade, confiabilidade, flexibilidade e custo [1]. O fator principal é que todas as técnicas trabalhem em sinergia produzindo produtos de acordo com o ritmo da demanda do cliente e com pouco ou nenhum desperdício. [2]

Em um mercado cada vez mais competitivo, como o automotivo, é fundamental que as empresas busquem essas características e apliquem de forma estratégica, ainda mais por se tratar do Brasil, país onde o segmento teve pouca visibilidade durante o início da história da indústria automobilística mundial e consequentemente se atrasou em relação a outros mercados. [3]

Assim, a Manufatura Enxuta pode auxiliar por ser um modelo estratégico e integrado de gestão, que visa o alto desempenho norteado por princípios e técnicas que suportam esse modelo. [4]

Mas nem sempre os conceitos por trás da manufatura enxuta são devidamente absorvidos e acabam sendo implementados, em alguns casos podemos identificar essa implementação dos conceitos de manufatura enxuta nas empresas de maneira vaga e superficial. Nesse cenário, o presente trabalho irá caracterizar as informações mais difundidas sobre as técnicas e metodologias enxutas, através de um questionário respondido por representantes de empresas e, assim, mapear quais as técnicas estão sendo aplicadas por completo. 
O objetivo deste trabalho é identificar o uso de técnicas específicas de manufatura enxuta no setor automotivo brasileiro. Para isto um referencial teórico foi desenvolvido. Com base no referencial teórico as principais técnicas de manufatura enxuta foram identificadas. Posteriormente, uma pesquisa de campo para identificar a prática destas técnicas no setor automotivo foi realizada. Os resultados desta pesquisa de campo são mostrados e discutidos e o trabalho é encerrado com as considerações finais.

\section{REFERENCIAL TEÓRICO}

A implantação dos ideais japoneses envolvidos na manufatura enxuta, como o sistema just in time e o kanban, ocorrida em meados de 1990, trouxe uma reestruturação produtiva ao mercado brasileiro. Tal transformação se fez necessária tendo em vista a concorrência intensa, que se mantém até os dias de hoje e que faz com que as técnicas lean tenham grande importância para a competitividade das empresas. [5]

A importância da manufatura enxuta não somente se faz necessária para alavancar os resultados financeiros, mas também se relacionam com o conceito da sustentabilidade, fator que impulsiona as vendas e cria maior ligação entre empresa e seus consumidores.

O atual nível competitivo leva diversos segmentos a procurar agudamente evitar desperdícios que não agreguem valor, reduzir os custos e agradar cada vez mais os clientes. Ainda como na época da reestruturação produtiva ocorrida nos anos 90, as técnicas lean são reconhecidas como a melhor alternativa para se alcançar essas vantagens. [6]

\subsection{Consolidação da pesquisa}

Na primeira etapa de desenvolvimento do referencial teórico, o objetivo foi identificar as principais técnicas de lean manufacturing publicadas nos últimos onze anos. Para isto pesquisou-se principalmente artigos e teses de doutorado sobre o tema tratado.

A Tabela 1 apresenta a pesquisa referente a artigos publicados nos últimos onze anos (2005 até 2015) que utilizam técnicas de manufatura enxuta. As bases de dados ScienceDirect e SpringerLink foram utilizadas aplicando palavra chave de busca "lean manufacturing", filtro de ano da publicação "2005 até 2015" e área "engineering e computer science". 
Tabela 1 - Tabela das técnicas de manufatura enxuta pesquisada em artigos científicos.

\begin{tabular}{|c|c|c|}
\hline $\begin{array}{l}\text { Autor (es) / Ano da } \\
\text { Publicação }\end{array}$ & Título do artigo & $\begin{array}{l}\text { Técnicas de manufatura enxuta } \\
\text { identificadas/citadas }\end{array}$ \\
\hline $\begin{array}{l}\text { SALEM, R; } \\
\text { MUSHARAVATI, F; } \\
\text { HAMOUDA, A.M; AL- } \\
\text { KHALIFA, K.N (2015) }\end{array}$ & $\begin{array}{c}\text { An empirical study on lean } \\
\text { awareness and potential for } \\
\text { lean } \\
\text { implementations in Qatar } \\
\text { industries }\end{array}$ & $\begin{array}{c}\text { Just in time (JIT) } \\
5 \text { S } \\
\text { Kanban } \\
\text { Fluxo de valor (VSM) } \\
\text { Manutenção Produtiva total (TPM) }\end{array}$ \\
\hline CHIARINI, A. (2014) & $\begin{array}{l}\text { Sustainable manufacturing- } \\
\text { greening processes using } \\
\text { specific Lean Production } \\
\text { tools: an empirical } \\
\text { observation from European } \\
\text { motorcycle } \\
\text { component manufacturers }\end{array}$ & $\begin{array}{c}\text { Fluxo de valor (VSM) } \\
5 \text { s } \\
\text { Cellular manufacturing } \\
\text { Single minute exchange of die (SMED) } \\
\text { Manutenção produtiva total (TPM) }\end{array}$ \\
\hline $\begin{array}{l}\text { JAMES, J; IKUMA, } \\
\text { L.H; NAHMENS, I; } \\
\text { AGHAZADEH, } \\
\text { F(2014) }\end{array}$ & $\begin{array}{l}\text { The impact of Kaizen on } \\
\text { safety in modular home } \\
\text { manufacturing }\end{array}$ & $\begin{array}{c}\text { Kaizen } \\
\text { Fluxo de valor (VSM) }\end{array}$ \\
\hline $\begin{array}{l}\text { JABBOUR, C, J; } \\
\text { JABBOUR, A, B, L, S; } \\
\text { GOVINDAN, K; } \\
\text { TEIXEIRA, A, A; } \\
\text { FREITAS, W, R, S. } \\
\text { (2013) }\end{array}$ & $\begin{array}{c}\text { Environmental management } \\
\text { and operational performance } \\
\text { in automotive companies in } \\
\text { Brazil: the role of human } \\
\text { resource } \\
\text { management and lean } \\
\text { manufacturing }\end{array}$ & $\begin{array}{c}5 \mathrm{~S} \\
\text { Kaizen } \\
\text { Just in time (JIT) } \\
\text { Kanban }\end{array}$ \\
\hline $\begin{array}{l}\text { BHASKARAN, E. } \\
(2012)\end{array}$ & $\begin{array}{l}\text { Lean Manufacturing Auto } \\
\text { Cluster at Chennai }\end{array}$ & $\begin{array}{c}5 S \\
\text { Kaizen } \\
\text { Single minute exchange of die (SMED) } \\
\text { Manutenção produtiva total (TPM) } \\
\text { Fluxo de valor (VSM) } \\
\text { Poka-yoke } \\
\text { Just in time (JIT) } \\
\text { Kanban } \\
\text { Six sigma }\end{array}$ \\
\hline $\begin{array}{l}\text { VINODH, S; ARVIND, } \\
\text { K.R; SOMANAATHAN, } \\
\text { M. (2011) }\end{array}$ & $\begin{array}{l}\text { Tools and techniques for } \\
\text { enabling sustainability } \\
\text { through lean initiatives }\end{array}$ & $\begin{array}{c}\text { Fluxo de valor (VSM) } \\
7 \text { S refere-se a } 5 S+2 S \text { (safety + sustainability) } \\
\text { Kaizen } \\
\text { Cellular manufacturing } \\
\text { Manutenção preventiva total (TPM) } \\
\text { Six Sigma } \\
\text { Poka-yoke }\end{array}$ \\
\hline
\end{tabular}




\begin{tabular}{|c|c|c|}
\hline $\begin{array}{c}\text { DOMBROWSKI, U; } \\
\text { CRESPO, I; ZAHN, T. } \\
\text { (2010) }\end{array}$ & $\begin{array}{l}\text { Adaptive Configuration of a } \\
\text { Lean Production System } \\
\text { in Small and Medium-sized } \\
\text { Enterprises }\end{array}$ & $\begin{array}{c}5 \mathrm{~s} \\
\text { Manutenção produtiva total (TPM) } \\
\text { Kaizen } \\
\text { Kanban } \\
\text { Just in time (JIT) }\end{array}$ \\
\hline $\begin{array}{c}\text { ÀLVAREZ, R; CALVO, } \\
\text { R; PENAA, M.M; } \\
\text { DOMINGO, R (2009) }\end{array}$ & $\begin{array}{l}\text { Redesigning an assembly } \\
\text { line through lean } \\
\text { manufacturing tools }\end{array}$ & $\begin{array}{c}\text { Fluxo de valor (VSM) } \\
\text { Kanban } \\
\text { Benchmarking }\end{array}$ \\
\hline $\begin{array}{c}\text { DAL PONT, G; } \\
\text { FURLAN, A; VINELLI, } \\
\text { A. (2008) }\end{array}$ & $\begin{array}{l}\text { Interrelationships among } \\
\text { lean bundles and their effects } \\
\text { on operational performance }\end{array}$ & $\begin{array}{l}\text { Poka-yoke } \\
\text { Just in time (JIT) } \\
\text { Kanban }\end{array}$ \\
\hline $\begin{array}{l}\text { ABDULMALEKA, F.A; } \\
\text { RAJGOPAL, J. (2007) }\end{array}$ & $\begin{array}{l}\text { Analyzing the benefits of lean } \\
\text { manufacturing and value } \\
\text { stream } \\
\text { mapping via simulation: A } \\
\text { process sector case study }\end{array}$ & $\begin{array}{c}5 S \\
\text { Kaizen } \\
\text { Manutenção produtiva total (TPM) } \\
\text { Just in time (JIT) } \\
\text { Kanban } \\
\text { Heijunka } \\
\text { Fluxo de valor (VSM) }\end{array}$ \\
\hline $\begin{array}{c}\text { CAKMAKCI, M; } \\
\text { KARASU, M. K. (2007) }\end{array}$ & $\begin{array}{c}\text { Set-up time reduction } \\
\text { process and integrated } \\
\text { predetermined time } \\
\text { system MTM-UAS: A study } \\
\text { of application in a large size } \\
\text { company } \\
\text { of automobile industry }\end{array}$ & $\begin{array}{c}\text { Manutenção produtiva total (TPM) } \\
\text { Just in time (JIT) }\end{array}$ \\
\hline $\begin{array}{l}\text { SHEN, S.X; HAN, C.F. } \\
(2006)\end{array}$ & $\begin{array}{l}\text { China electrical } \\
\text { manufacturing services } \\
\text { industry value } \\
\text { stream mapping } \\
\text { collaboration }\end{array}$ & Fluxo de valor (VSM) \\
\hline MELTON, T. (2005) & $\begin{array}{l}\text { The Benefits of Lean } \\
\text { Manufacturing: What Lean } \\
\text { Thinking has to Offer the } \\
\text { Process Industries }\end{array}$ & $\begin{array}{c}5 S \\
\text { Kaizen } \\
\text { Kanban } \\
\text { Poka-yoke } \\
\text { Fluxo de valor (VSM) }\end{array}$ \\
\hline
\end{tabular}

Na segunda etapa buscou-se teses de doutorado publicadas no período de 2006 até 2014. As bases de dados de teses das universidades USP, UFSC, UNESP, UNICAMP, UFSCAR foram utilizadas aplicando palavra chave de busca "Manufatura enxuta" e filtro de ano da publicação “2006 até 2014”. A tabela 2 mostra uma consolidação das teses consultadas. 
Tabela 2 - Tabela das técnicas de manufatura enxuta pesquisada em teses.

\begin{tabular}{|c|c|c|}
\hline $\begin{array}{l}\text { Autor(es) / Ano da } \\
\text { Publicação }\end{array}$ & Título do artigo & $\begin{array}{c}\text { Técnicas de manufatura enxuta } \\
\text { identificadas/citadas }\end{array}$ \\
\hline $\begin{array}{l}\text { MACHADO, M.C } \\
(2006)\end{array}$ & $\begin{array}{c}\text { Princípios enxutos no } \\
\text { processo de } \\
\text { desenvolvimento de } \\
\text { produtos: proposta de uma } \\
\text { metodologia para } \\
\text { implementação } \\
\end{array}$ & $\begin{array}{c}\text { Fluxo de valor (VSM) } \\
\text { Just in Time (JIT) } \\
\text { Kanban } \\
\text { Kaizen }\end{array}$ \\
\hline $\begin{array}{l}\text { ANDRADE, G.J.P.O. } \\
\qquad(2006)\end{array}$ & $\begin{array}{l}\text { Um método de diagnóstico } \\
\text { do potencial da aplicação da } \\
\text { manufatura enxuta na } \\
\text { indústria textil }\end{array}$ & $\begin{array}{c}\text { Manutenção Produtiva Total (TPM) } \\
\text { Just in Time (JIT) } \\
\text { Kanban } \\
\text { Fluxo de valor (VSM) } \\
\text { Benchmarking }\end{array}$ \\
\hline MUNIZ JR, J. (2007) & $\begin{array}{l}\text { Modelo conceitual de gestão } \\
\text { de produção baseado na } \\
\text { gestão do conhecimento: um } \\
\text { estudo no ambiente operário } \\
\text { da indústria automotiva }\end{array}$ & $\begin{array}{c}\text { Just in time (JIT) } \\
\text { Kaizen } \\
5 \mathrm{~S} \\
\text { Poka-yoke }\end{array}$ \\
\hline $\begin{array}{l}\text { MAGALHÃES, J.J.L. } \\
(2007)\end{array}$ & $\begin{array}{l}\text { O emprego do custo meta } \\
\text { em uma estrutura de } \\
\text { consórcio modular: um } \\
\text { estudo multicaso para os } \\
\text { sistemas do consórcio } \\
\text { modular da FORD de } \\
\text { Camaçari/BA }\end{array}$ & Kaizen \\
\hline $\begin{array}{l}\text { ESPOSTO, K.F. } \\
\qquad(2008)\end{array}$ & $\begin{array}{c}\text { Elementos estruturais para } \\
\text { gestão de desempenho em } \\
\text { ambientes de produção } \\
\text { enxuta }\end{array}$ & $\begin{array}{c}\text { Kaizen } \\
\text { Just in time (JIT) } \\
\text { Fluxo de valor (VSM) } \\
5 \text { S } \\
\text { Manutenção Produtiva Total (TPM) } \\
\text { Poka-yoke } \\
\text { Kanban } \\
\text { SMED }\end{array}$ \\
\hline SILVA, A.S.F. (2009) & $\begin{array}{c}\text { Avaliação de práticas e } \\
\text { performance da manufatura } \\
\text { enxuta, via benchmarking, } \\
\text { para diagnóstico da indústria } \\
\text { de confecções. }\end{array}$ & $\begin{array}{c}\text { Fluxo de valor (VSM) } \\
\text { Benchmarking } \\
\text { Just in time (JIT) } \\
\text { Kanban } \\
\text { Manutenção Produtiva Total (TPM) }\end{array}$ \\
\hline $\begin{array}{l}\text { ARAUJO, C.A.C. } \\
(2010)\end{array}$ & $\begin{array}{c}\text { Estudo de causas e } \\
\text { estratégias para lidar com } \\
\text { variação na utilização da } \\
\text { capacidade dos recursos } \\
\text { produtivos em ambientes de } \\
\text { empresas enxutas }\end{array}$ & $\begin{array}{c}\text { Heijunka } \\
\text { Kanban } \\
\text { Six Sigma } \\
\text { Fluxo de valor (VSM) }\end{array}$ \\
\hline
\end{tabular}




\begin{tabular}{|c|c|c|}
\hline $\begin{array}{l}\text { TORRES, L.F. } \\
(2011)\end{array}$ & $\begin{array}{c}\text { Avaliação da aplicação da } \\
\text { manufatura enxuta em uma } \\
\text { empresa do setor } \\
\text { automobilístico: um estudo } \\
\text { de caso }\end{array}$ & $\begin{array}{c}\text { Just in time (JIT) } \\
\text { Fluxo de valor (VSM) } \\
\text { SMED } \\
\text { Kanban }\end{array}$ \\
\hline $\begin{array}{l}\text { LOURENÇO JR, J. } \\
\text { (2012) }\end{array}$ & $\begin{array}{l}\text { A produção enxuta em um } \\
\text { sistema de fabricação } \\
\text { contínuo: aplicação da } \\
\text { simulação discreta } \\
\text { estocástica na indústria de } \\
\text { condutores elétricos. }\end{array}$ & $\begin{array}{c}\text { Fluxo de valor (VSM) } \\
\text { Just in time (JIT) } \\
\text { Kanban }\end{array}$ \\
\hline $\begin{array}{c}\text { MATEUS JR, J.R. } \\
(2013)\end{array}$ & $\begin{array}{c}\text { Modelo de gestão da } \\
\text { ergonomia integrado as } \\
\text { práticas da produção enxuta } \\
\text { - ERGOPRO: o caso de uma } \\
\text { empresa de embalagem de } \\
\text { papelão ondulado }\end{array}$ & $\begin{array}{c}5 S \\
\text { Kaizen } \\
\text { Heijunka } \\
\text { Fluxo de valor (VSM) } \\
\text { SMED } \\
\text { Kanban }\end{array}$ \\
\hline $\begin{array}{l}\text { LAZARIN, D.F. } \\
\text { (2013) }\end{array}$ & $\begin{array}{l}\text { Estratégia de produção e a } \\
\text { abordagem da manufatura } \\
\text { enxuta: estudos de caso no } \\
\text { setor de autopeças brasileiro }\end{array}$ & $\begin{array}{c}\text { Fluxo de valor (VSM) } \\
\text { Kaizen } \\
5 S \\
\text { Kanban } \\
\text { Manutenção produtiva total (TPM) } \\
\text { Just in time (JIT) } \\
\text { Poka-yoke }\end{array}$ \\
\hline $\begin{array}{l}\text { RENÓ, G.W.S. } \\
\text { (2014) }\end{array}$ & $\begin{array}{l}\text { Trabalho padronizado e } \\
\text { equipes semi autônomas: } \\
\text { adaptação, aplicação e } \\
\text { análise de um modelo a } \\
\text { partir de múltiplos casos em } \\
\text { uma empresa do setor } \\
\text { químico }\end{array}$ & $\begin{array}{c}\text { Just in time (JIT) } \\
\text { Fluxo de valor (VSM) } \\
\text { Kanban } \\
5 S \\
\text { Manutenção produtiva total (TPM) }\end{array}$ \\
\hline
\end{tabular}

Para a análise dos dados da pesquisa realizada em artigos (Tabela 1) e teses (Tabela 2) e consolidação do resultado foi utilizado o Diagrama de Pareto, que consiste em um gráfico de colunas que ordena as frequências das aparições das técnicas de manufatura enxuta do maior para o menor, dessa forma foi possível identificar quais as técnicas mais citadas nos artigos e teses.

Foi utilizado o limite de $80 \%$ da frequência acumulada (eixo secundário dos gráficos) como critério de corte para identificar as técnicas mais utilizadas. Dessa forma através das Figura 1 e 2 foi possível concluir que Fluxo de Valor (VSM), 5S, Kanban, Just in Time (JIT), Manutenção Produtiva Total (TPM) e Kaizen foram as técnicas mais citadas em ambos os casos. 


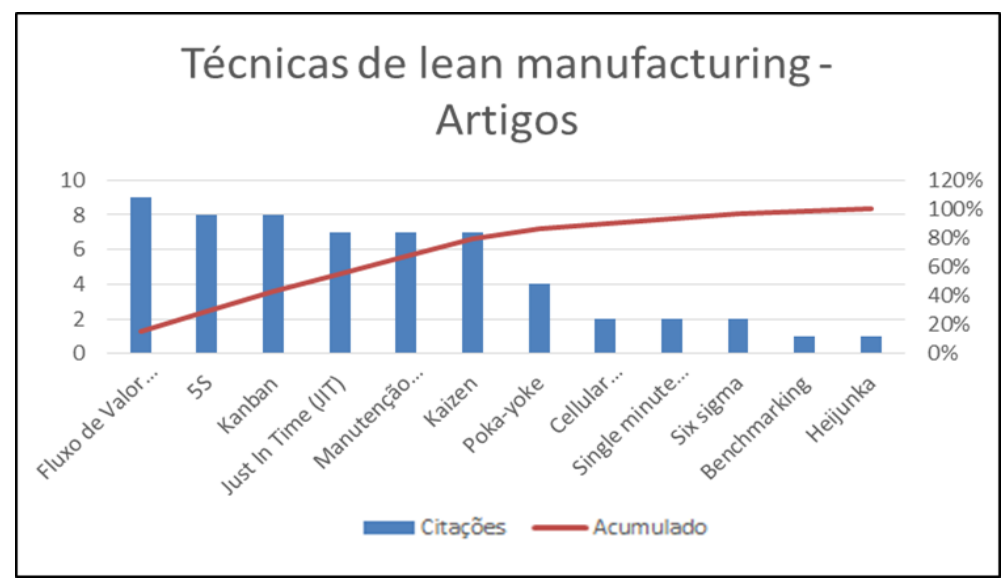

Figura 1: Gráfico de Pareto dos artigos.

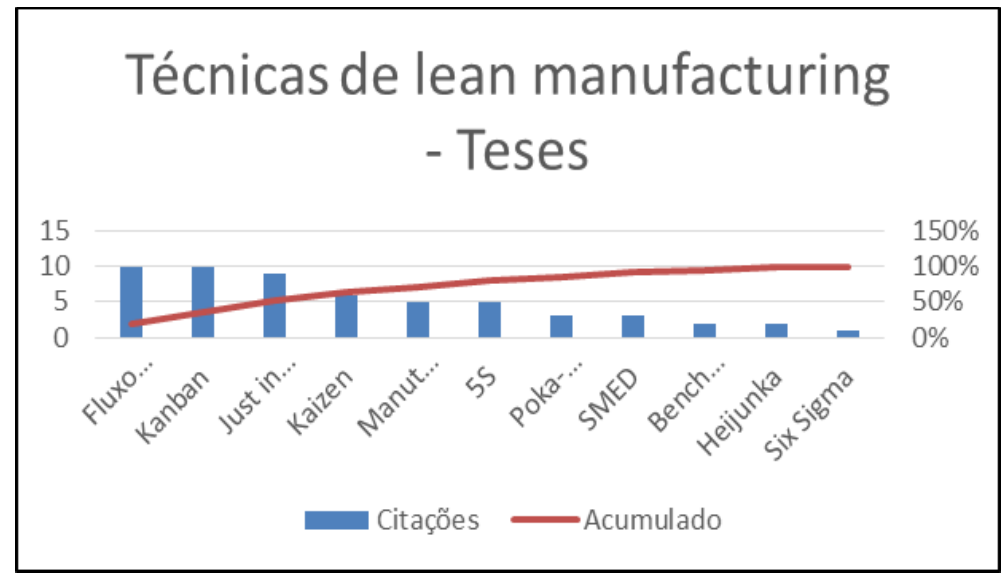

Figura 2: Gráfico de Pareto das teses.

Com a análise dos gráficos expostos nas figuras 1 e 2 foi possível identificar que seis técnicas se destacam na consolidação das técnicas utilizadas. $\mathrm{O}$ critério para selecionar as técnicas que seriam perguntadas no questionário se deu através da quantidade de citações apresentadas nas tabelas, isto é, foi utilizado como critério de corte a aparição de pelo menos 6 vezes da técnica, portanto na Tabela 1 identificamos 13 artigos e as técnicas que apareceram em pelo menos 6 artigos foram: Fluxo de valor total (TPM), 5S, Kanban, Just in time (JIT), Manutenção Produtiva Total (TPM) e Kaizen. Já na Tabela 2 identificamos 12 teses e seguindo o mesmo critério as técnicas selecionadas foram: Fluxo de valor (VSM), Kanban, Just in time (JIT) e Kaizen. Portanto através dessa análise o questionário foi elaborado com as quatro técnicas presentes nos dois casos (VSM, Kanban, Kaizen, JIT).

A partir dessas informações foi elaborado o questionário que teve como preocupação ser construído de maneira que as respostas fossem objetivas. Cada técnica teve suas atividades principais descritas, evitando assim, respostas genéricas. Para facilitar o entendimento da importância de cada técnica também foi proposta a classificação do grau de significância.

A análise do questionário foi feita estatisticamente através dos dados quantitativos levantados e com o auxílio de dois gráficos: um gráfico de pizza que mostra a porcentagem de respostas entre "sim", "não" e "não sei" para a aplicação de cada atividade dentro da sua respectiva técnica e outro que contabilizou a quantidade de respostas sobre o grau de importância da 
atividade em questão. Com isso foi possível observar estatisticamente a relevância de cada técnica.

\section{PESQUISA DE CAMPO}

A pesquisa de campo foi realizada por meio de um questionário que contém perguntas elaboradas a partir das citações das técnicas lean consolidadas.

O questionário foi estruturado em três seções:

- Informações sobre o respondente.

- Questionário.

- Outras técnicas de manufatura enxuta.

A segunda secção está estruturada em duas perguntas, a primeira pergunta (Sua empresa pratica?) consiste em identificar a prática da atividade na empresa em que o respondente trabalha. O respondente deve escolher como resposta apenas uma das três alternativas possíveis: "Sim", "Não" ou "Não sei", a segunda pergunta (Qual a importância da atividade?) consiste em avaliar, de acordo com a opinião do respondente, a importância da atividade considerando seu custo/benefício. O respondente deve escolher como resposta apenas uma das cinco alternativas possíveis: "1", "2", "3", "4" ou "5".

Ao final do questionário encontra-se a secção OUTRAS TÉCNICAS DE MANUFATURA ENXUTA para que o respondente descreva livremente demais técnicas não citadas nesse questionário, contudo praticadas na empresa em que o respondente trabalha.

As perguntas do questionário serão específicas com o objetivo de extrair informações necessárias para a análise, após coletar os dados do nosso público alvo será realizada uma seleção das informações relevantes para que seja possível apresentar os dados de maneira estatística.

O intuito é qualificar se as técnicas estão sendo aplicadas completamente, parcialmente ou não estão sendo aplicadas nas empresas pesquisadas.

\section{ANÁLISE E DESCUSSÃO DE RESULTADOS}

\subsection{Amostra da pesquisa}

$\mathrm{O}$ foco da pesquisa foi encontrar respondentes que tinham contato direto e conhecimentos dos processos produtivos em sua respectiva empresa. A amostra selecionada foi composta de 22 pessoas todos com pelo menos 1 ano de trabalho na empresa, a média de tempo de trabalho foi de aproximadamente 6 anos.

A amostra selecionada foi composta por profissionais envolvidos com a manufatura enxuta. Analisando a classificação das empresas na Figura 3 observa-se que a amostra foi predominantemente composta por profissionais de montadoras de veículos do setor automotivo brasileiro. 


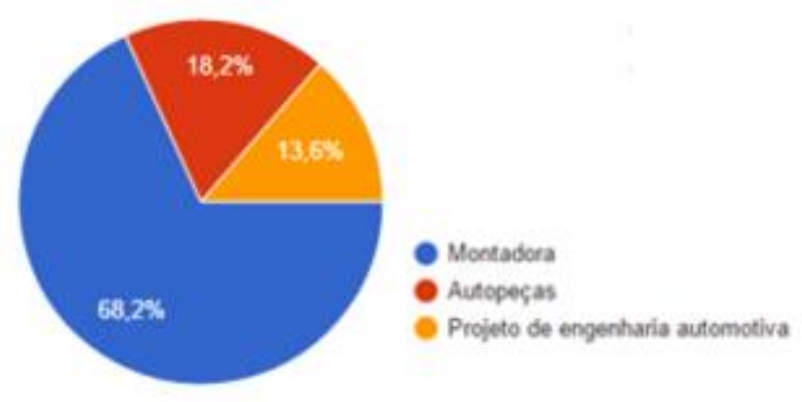

Figura 3: Classificação da amostra

\subsection{Mapeamento do fluxo de valor}

A técnica de manufatura enxuta Mapeamento do fluxo de valor em suas 3 atividades pelo menos $77 \%$ dos participantes da pesquisa respondeu com "Sim" quando questionado se a empresa pratica tal atividade. Podemos destacar que todas as atividades tiveram predominância na classificação como "Muito Importante", isto é, a grande maioria na escala de 1 (não importante) a 5 (muito importante) informaram o número 5 demonstrando que as atividades são essenciais para otimizar a performance da empresa (Figura 4).

Dentre as 3 atividades, a "Definir os fluxos (materiais e informações) do processo, determinando os tempos de cada atividade e suas sequências" foi a que as empresas mais aplicam, onde quase todos da amostra responderam "Sim" quando questionado se a empresa pratica. 


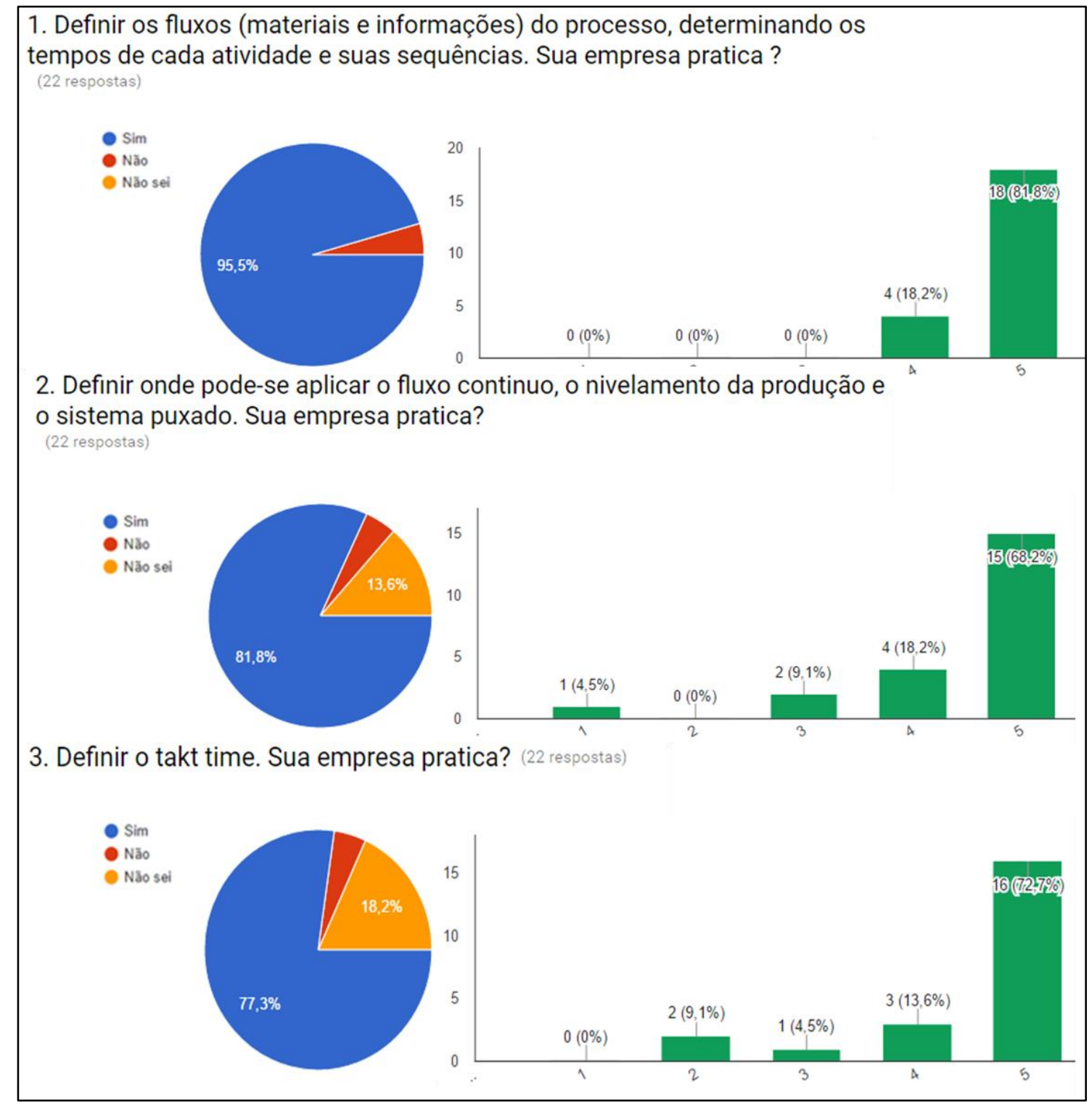

Figura 4: Atividades de Mapeamento do Fluxo de valor.

\subsection{Kanban}

A técnica de manufatura enxuta Kanban em suas 3 atividades pelo menos $72 \%$ dos participantes da pesquisa respondeu com "Sim" quando questionado se a empresa pratica tal atividade. Podemos destacar que somente a atividade "Informar fornecedor para envio de novas peças" teve a predominância na classificação como "Muito Importante", isto é, a grande maioria na escala de 1 (não importante) a 5 (muito importante) informaram o número 5 , as demais atividades tiveram equilíbrio nos números 3,4 e 5 da escala tornando inconclusivo se é essencial tal atividade (Figura 5).

Dentre as 3 atividades, a "Armazenar material em locais intermediários para posterior envio ao destino final" e "Informar fornecedor para envio de novas peças" foram a que as empresas mais aplicam, onde quase todos da amostra responderam "Sim" quando questionado se a empresa pratica. 


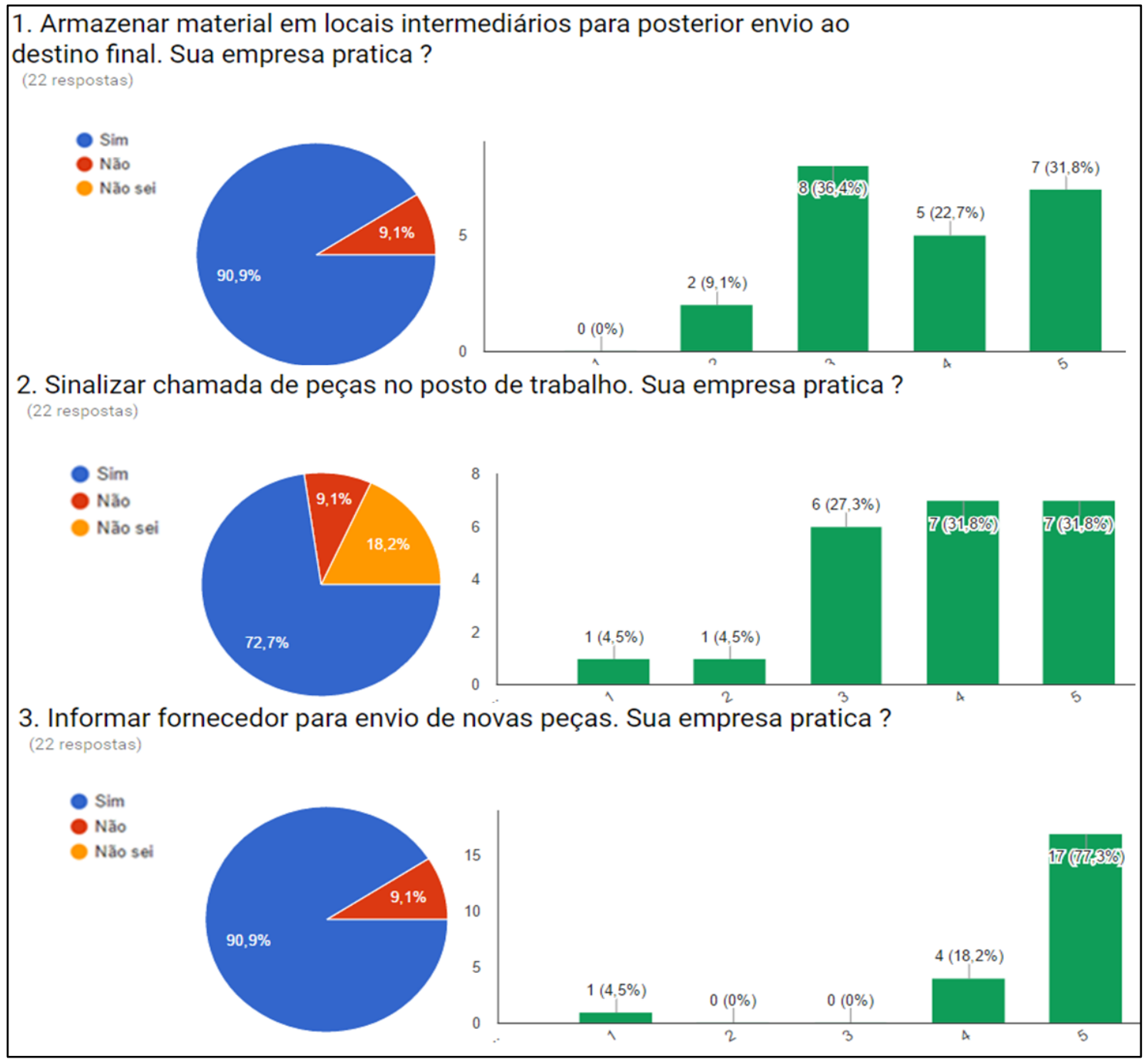

Figura 5: Atividades de Kanban.

\subsection{Just in time (JIT)}

A técnica de manufatura enxuta Just in time em suas 5 atividades pelo menos $86 \%$ dos participantes da pesquisa respondeu com "Sim" quando questionado se a empresa pratica tal atividade. Podemos destacar que todas as atividades tiveram predominância na classificação como "Muito Importante", isto é, a grande maioria na escala de 1 (não importante) a 5 (muito importante) informaram o número 5 demonstrando que as atividades são essenciais para otimizar a performance da empresa (Figura 6).

Dentre as 3 atividades, a "Eliminar desperdícios como as atividades que consomem recursos e não agregam valor" todos da amostra responderam "Sim" quando questionado se a empresa pratica, e JIT foi a que teve a melhor performance de todas as técnicas analisadas na pesquisa, isto é, todas as atividades foram classificadas predominantemente como muito importante e obteve maior presença em porcentagem de aplicação das atividades. 
1. Eliminar desperdícios como as atividades que consomem recursos e não agregam valor. Sua empresa pratica?

(22 respostas)

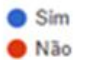

- Não

- Não sei
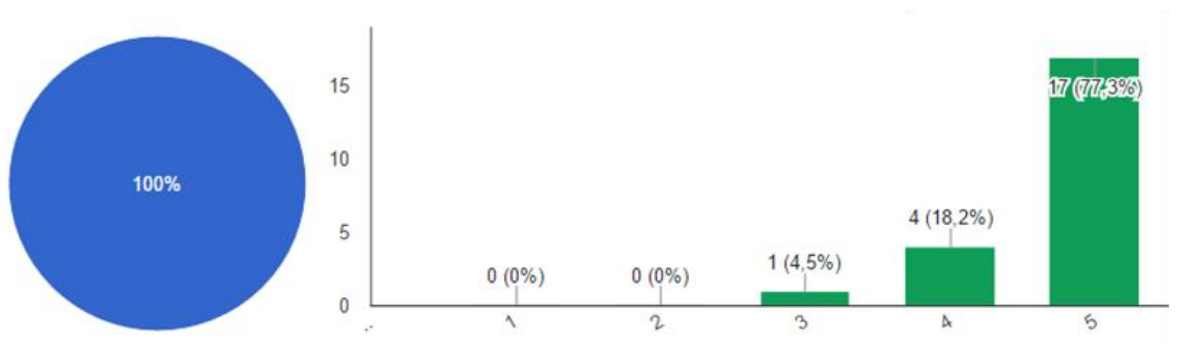

2. Reduzir estoques. Sua empresa pratica? (22 respostas)

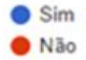

Nào sei
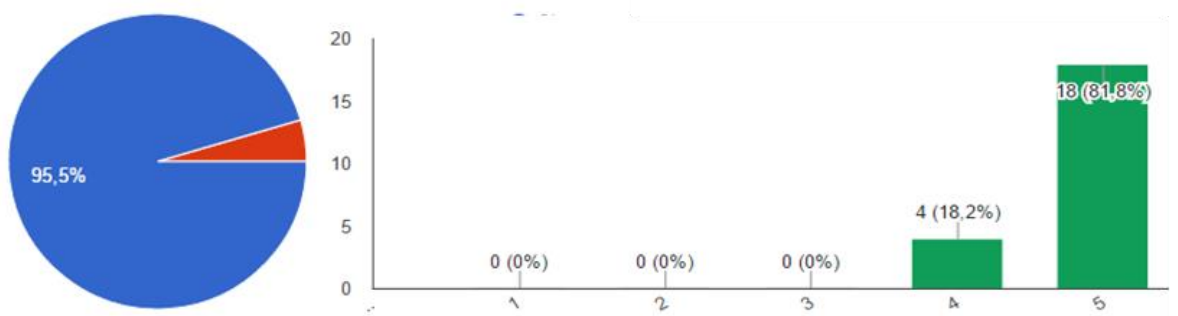

3. Reduzir transporte interno. Sua empresa pratica? (22 respostas)
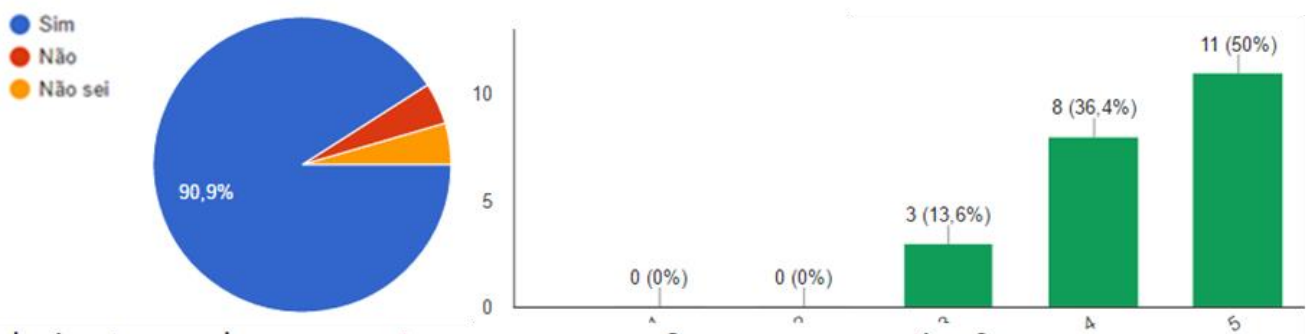

4. Reduzir o tempo de espera entre os processos. Sua empresa pratica? (22 respostas)
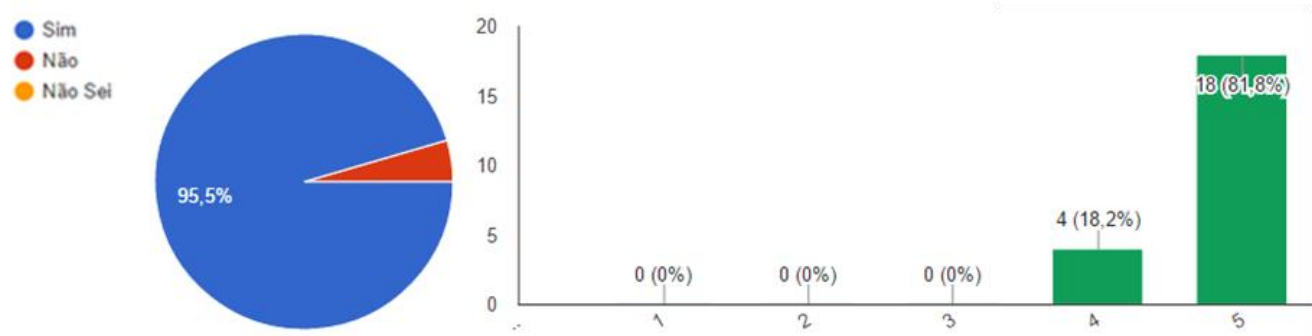

5. Eliminar refugos de trabalho. Sua empresa pratica? (22 respostas)

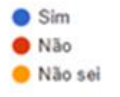

- Nåo sei

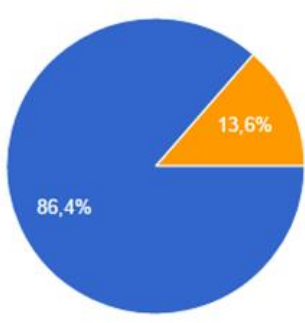

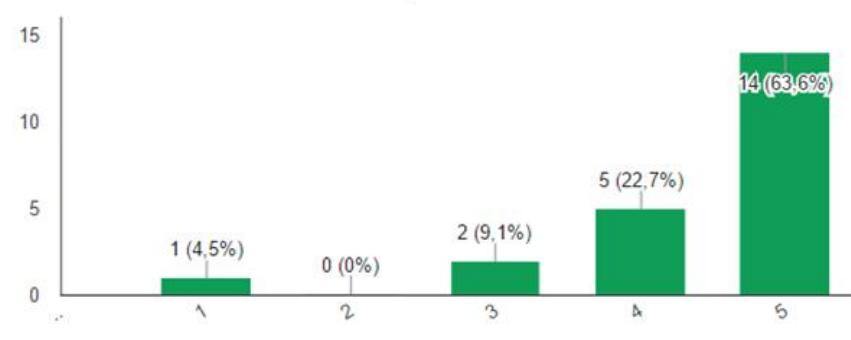

Figura 6: Atividades de Just in time (JIT). 


\subsection{Kaizen}

A técnica de manufatura enxuta Kaizen em suas 3 atividades pelo menos $77 \%$ dos participantes da pesquisa respondeu com "Sim" quando questionado se a empresa pratica tal atividade. Podemos destacar que todas as atividades tiveram predominância na classificação como "Muito Importante", isto é, a grande maioria na escala de 1 (não importante) a 5 (muito importante) informaram o número 5 demonstrando que as atividades são essenciais para otimizar a performance da empresa (Figura 7).

Dentre as 3 atividades, a "Desenvolver ações que visem eliminar desperdícios no chão-defábrica e melhorem o conforto e a segurança no trabalho" foi a que as empresas mais aplicam, onde quase todos da amostra responderam "Sim" quando questionado se a empresa pratica.

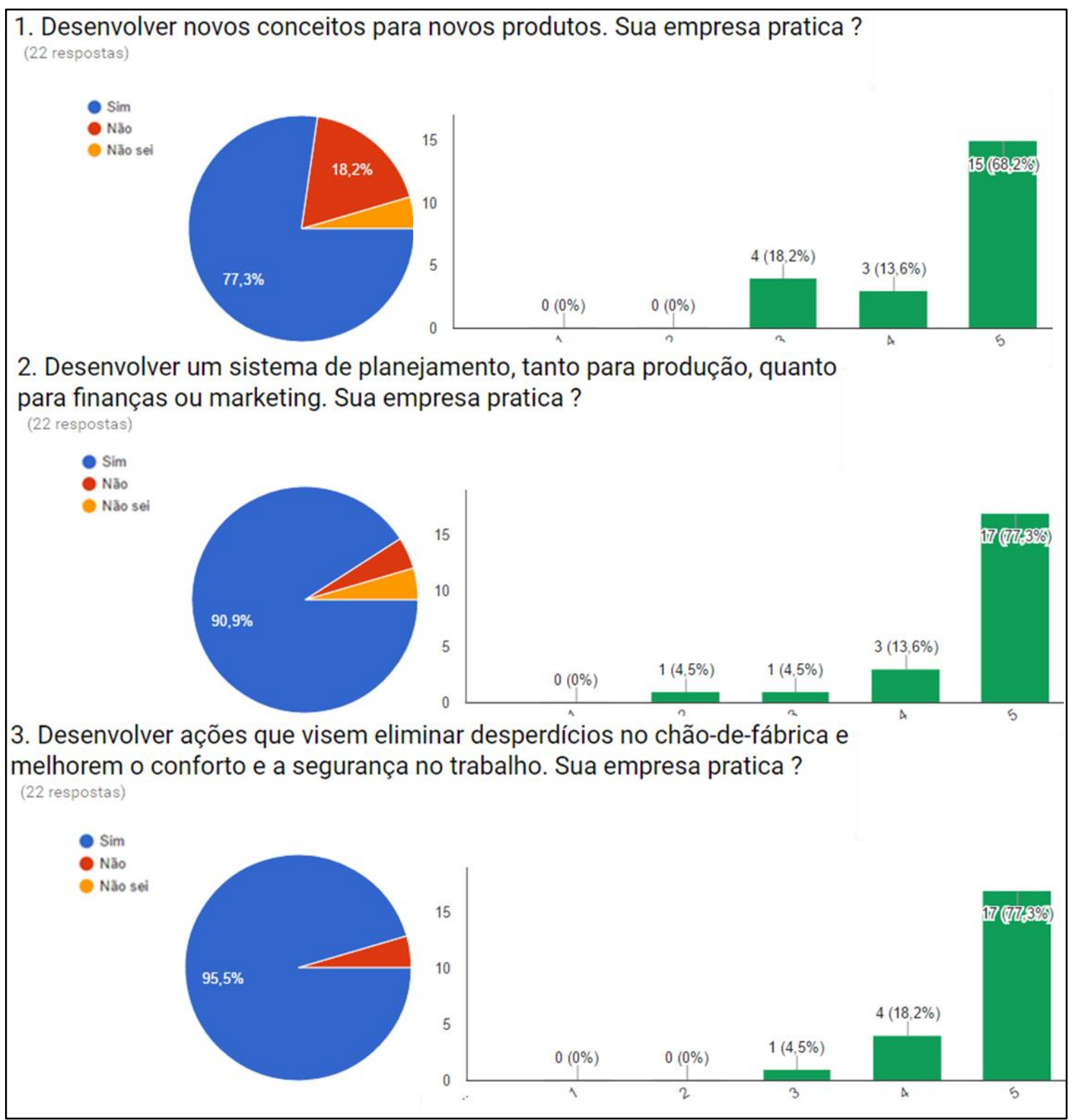

Figura 7: Atividades de Kaizen. 


\subsection{Outras técnicas lean}

Com o objetivo de verificar quais outras técnicas de manufatura enxuta são aplicadas nas empresas da amostra, o questionário tinha uma secção para o respondente citar a técnicas e seu respectivo grau de importância. Analisando toda a amostra encontramos diversas técnicas que são utilizadas, porém vale destacar as que foram classificadas com o grau máximo de importância: 5S, 8 disciplinas, Espinha de peixe, 6 Sigma, PJO - Process Job Observation, 5 PQ`s, indicadores QCDM - Levantamento e trativa de qualidade das peças/custo/entrega/motivação dos envolvidos.

\section{CONCLUSÃO}

Conclui-se que no geral as técnicas lean estão sendo aplicadas, sendo sua importância apontada como muito importante na maioria das situações, sugerindo que as empresas estão alinhadas com as tendências e competitividade. Para as quatro técnicas selecionadas para o questionário (VSM, Kanban, Kaizen, JIT), houve respostas positivas em todas, sinalizando um bom desempenho das empresas brasileiras nos segmentos propostos.

Outro destaque da pesquisa foi a importância dada as técnicas de manufatura enxuta e as atividades relacionadas a elas, sinalizando uma expectativa positiva quanto a continuidade da aplicação das mesmas.

Quanto as outras técnicas lean (5S, 8 disciplinas, Espinha de peixe, 6 Sigma, PJO - Process Job Observation, 5 PQ`s, indicadores QCDM) mencionadas nos resultados e que tiveram grau máximo de importância, percebe-se que na prática são classificadas como muito importante, quando na revisão em teses e artigos tem menor visibilidade.

Para trabalhos futuros recomenda-se expandir a pesquisa para micro e pequenas empresas automotivas, e assim, obter um maior panorama do uso das técnicas de lean manufacturing no setor automotivo brasileiro.

\section{REFERENCIAS}

[1] SLACK, N.; CHAMBERS, S.; JOHNSTON, R. Administração da Produção. $3^{a}$ ed., Atlas, São Paulo, 2009

[2] SHAH, R; WARD, P. T. Leanmanufacturing: context, practicebundles, andperformance. JournalofOperations Management, v. 21, n. 2, p. 129-149, mar. 2003.

[3] WOMACK, J. P.; JONES, D. T.; ROOS, D.; (1992). A Máquina que mudou o mundo, Campus, Rio de Janeiro, 2004.

[4] GODINHO FILHO, M.; FERNANDES, F.C.F. Manufatura Enxuta: uma revisão que classifica e analisa os trabalhos apontando perspectivas de pesquisas futuras, Gestão e Produção, São Carlos, 2004.

[5] ANTUNES, Ricardo. Os caminhos da liofilização organizacional: as formas diferenciadas da reestruturação produtiva no Brasil. Ideias (Unicamp), Campinas, v.9, n.2, v.10, n.1, p.13-24, 2003 
[6] ELIAS, Sérgio José Barbosa; MAGALHÃES, Liciane Carneiro. Contribuição da produção enxuta para obtenção da produção mais limpa. In: Encontro Nacional de Engenharia de Produção (Enegep), 23, 2003, Ouro Preto. Anais... Porto Alegre: Abepro, 2003.

[7] SALEM, R; MUSHARAVATI, F; HAMOUDA, A.M; AL-KHALIFA, K.N. An empirical study on lean awareness and potential for lean implementations in Qatar industries. The International Journal of Advanced Manufacturing Technology, London, July, 2015. pp 119.

[8] CHIARINI, A. Sustainable manufacturing-greening processes using specific Lean Production tools: na empirical observation from European motorcycle component manufacturers. Journal of Cleaner Production,15 December 2014. Volume 85, Pages 226233.

[9] JAMES, J; IKUMA, L.H; NAHMENS, I; AGHAZADEH, F. The impact of Kaizen on safety in modular home manufacturing. The International Journal of Advanced Manufacturing Technology, January, 2014. Volume 70, Issue 1-4, pp 725-734.

[10] JABBOUR, C, J; JABBOUR, A, B, L, S; GOVINDAN, K; TEIXEIRA, A, A; FREITAS, $\mathrm{W}, \mathrm{R}, \mathrm{S}$. Environmental management and operational performance in automotive companies in Brazil: the role of human resource management and lean manufacturing. Journal of Cleaner Production, May, 2013. Volume 47, Pages 129-140.

[11] BHASKARAN, E. Lean Manufacturing Auto Cluster at Chennai. Journal of The Institution of Engineers (India): Series C, October, 2012. Volume 93, Issue 4, pp 383-390.

[12] VINODH, S; ARVIND, K.R; SOMANAATHAN, M. Tools and techniques for enabling sustainability through lean initiatives. Clean Technologies and Environmental Policy, June 2011. Volume 13, Issue 3, pp 469-479.

[13] DOMBROWSKI, U; CRESPO, I; ZAHN, T. Adaptive Configuration of a Lean Production System in Small and Medium-sized Enterprises. Production Engineering, August 2010. Volume 4, Issue 4, pp 341-348.

[14] ÀLVAREZ, R; CALVO, R; PEÑA, M.M; DOMINGO, R. Redesigning an assembly line through lean manufacturing tools. The International Journal of Advanced Manufacturing Technology, August 2009. Volume 43, Issue 9-10, pp 949-958.

[15] DAL PONT, G; FURLAN, A; VINELLI, A. Interrelationships among lean bundles and their effects on operational performance. Operations Management Research, December 2008. Volume 1, Issue 2, pp 150-158.

[16] ABDULMALEKA, F.A; RAJGOPAL, J. Analyzing the benefits of lean manufacturing and value stream mapping via simulation: A process sector case study. International Journal of Production Economics, May 2007. Volume 107, Issue 1, Pages 223-236.

[17] CAKMAKCI, M; KARASU, M. K. Set-up time reduction process and integrated predetermined time system MTM-UAS: A study of application in a large size company of automobile industry. The International Journal of Advanced Manufacturing Technology, June 2007. Volume 33, Issue 3, pp 334-344.

[18] SHEN, S.X; HAN, C.F. China electrical manufacturing services industry value stream mapping collaboration. International Journal of Flexible Manufacturing Systems, December 2006. Volume 18, Issue 4, pp 285-303.

[19] MELTON, T. The Benefits of Lean Manufacturing: What Lean Thinking has to Offer the Process Industries. Chemical Engineering Research and Design, June 2005. Volume 83, Issue 6, Pages 662-673. 
[20] MACHADO, M.C. Princípios enxutos no processo de desenvolvimento de produtos: proposta de uma metodologia para implementação. 2006. 248f.. Tese (Doutorado) Departamento de Engenharia de Produção, Escola Politécnica da Universidade de São Paulo, São Paulo, 2006.

[21] ANDRADE, G.J.P.O. Um método de diagnóstico do potencial da aplicação da manufatura enxuta na indústria textil. 2006. 298f.. Tese (Doutorado) - Engenharia de Produção, Universidade Federal de Santa Catarina, Santa Catarina, 2006.

[22] MUNIZ JR, J. Modelo conceitual de gestão de produção baseado na gestão do conhecimento: um estudo no ambiente operário da indústria automotiva. 2007. 148F.. Tese (Doutorado em Engenharia Mecânica) - Faculdade de Engenharia do Campus de Guaratinguetá, Universidade Estadual Paulista, São Paulo, 2007.

[23] MAGALHÃES, J.J.L. O emprego do custo meta em uma estrutura de consórcio modular: um estudo multicaso para os sistemas do consórcio modular da FORD de Camaçari/BA. 2007. 172f.. Tese (Doutorado em Engenharia de Produção) - Programa de Pós-Graduação em Engenharia de Produção, Universidade Federal de Santa Catarina, Santa Catarina, 2007.

[24] ESPOSTO, K.F. Elementos estruturais para gestão de desempenho em ambientes de produção enxuta. 2008. 244f.. Tese (Doutorado) - Programa de Pós-Graduação e Área de Concentração em Engenharia de Produção, Escola de Engenharia de São Carlos da Universidade de São Paulo, São Paulo, 2008.

[25] SILVA, A.S.F. AVALIAÇÃO DE PRÁTICAS E PERFORMANCE DA MANUFATURA ENXUTA, VIA BENCHMARKING, PARA DIAGNÓSTICO DA INDÚSTRIA DE CONFECÇÕES. 2009. 229f.. Tese (Doutorado) - Programa de PósGraduação em Engenharia de Produção, Universidade Federal de Santa Catarina, Santa Catarina, 2009.

[26] ARAUJO, C.A.C. Estudo de causas e estratégias para lidar com variação na utilização da capacidade dos recursos produtivos em ambientes de empresas enxutas. 2010. 236f.. Tese (Doutorado) - Programa de Pós-Graduação em Engenharia de Produção e Área de Concentração Processos e gestão de operações, Escola de Engenharia de São Carlos da Universidade de São Paulo, São Paulo, 2010.

[27] TORRES, L.F. Avaliação da aplicação da manufatura enxuta em uma empresa do setor automobilístico: um estudo de caso. 2011. 123f.. Dissertação (Mestrado em Engenharia Mecânica) - Faculdade de Engenharia Mecânica, Universidade Estadual de Campinas, São Paulo, 2011.

[28] LOURENÇO JR, J. A PRODUÇÃO ENXUTA EM UM SISTEMA DE FABRICAÇÃO CONTÍNUO: APLICAÇÃO DA SIMULAÇÃO DISCRETA ESTOCÁSTICA NA INDÚSTRIA DE CONDUTORES ELÉTRICOS. 2012. 133f.. Tese (Doutorado) - Faculdade de Engenharia do Campus de Guaratinguetá, Universidade Estadual Paulista, São Paulo, 2012.

[29] MATEUS JR, J.R. Modelo de gestão da ergonomia integrado as práticas da produção enxuta - ERGOPRO: o caso de uma empresa de embalagem de papelão ondulado. 2013. 171f.. Tese (Doutorado) - Programa de Pós-Graduação em Engenharia de Produção, Universidade Federal de Santa Catarina, Santa Catarina, 2013.

[30] LAZARIN, D.F. Estratégia de produção e a abordagem da manufatura enxuta: estudos de caso no setor de autopeças brasileiro. 2013. 251f.. Tese (Doutorado) - Programa de Pós-Graduação em Engenharia de Produção, Universidade Federal de São Carlos, São Paulo, 2013. 
[31] RENÓ, G.W.S. Trabalho padronizado e equipes semi autônomas: adaptação, aplicação e análise de um modelo a partir de múltiplos casos em uma empresa do setor químico. 2014. 255f.. Tese (Doutorado) - Programa de Pós-Graduação em Engenharia de Produção, Universidade Federal de São Carlos, São Paulo, 2014. 